\title{
A Quaternion-Based Approach to Interference Alignment with Alamouti Coding
}

\author{
J. Fanjul, I. Santamaria, C. Loucera \\ Departamento de Ingeniera de Comunicaciones (DICOM) \\ Universidad de Cantabria \\ Plaza de la Ciencia, Santander, 39005, Spain \\ Email: \{fanjulj, i.santamaria, carlos.loucera \}@unican.es
}

\begin{abstract}
Based on the representation of Alamouti space-time codewords as quaternions, this paper proposes a scheme that combines interference alignment (IA) with Alamouti signals. The proposed formulation allows for a separation of the space-time block coding (to gain spatial diversity) and the IA precoding (to reduce or ideally suppress interference). Although this separation is not necessarily optimal, the splitting of alignment precoding and Alamouti encoding is particularly convenient because it enables the independent optimization of the IA solution using quaternionic versions of standard alternating optimization techniques such as the maximum signal-tointerference-plus-noise algorithm. Some numerical simulations are included to compare the performance of the proposed quaternion IA+Alamouti algorithm with standard IA algorithms in the complex domain as well as with interference cancellation schemes at the receiver side.

Index Terms-Interference alignment, MIMO interference channel, space-time block coding, Alamouti, quaternions.
\end{abstract}

\section{INTRODUCTION}

Interference alignment (IA) has received a lot of attention in recent years as a key technique to achieve the maximum degrees of freedom (DoF) of wireless networks in the presence of interference [1]. However, most of the IA schemes and algorithms proposed in the literature [2], [3] do not consider the reliability in terms of spatial diversity gain, which is another key performance metric.

Space-time block codes (STBCs), such as the popular Alamouti code designed assuming 2 antennas at the transmitter side [4], are simple linear processing schemes for achieving spatial diversity. Therefore, it is of interest to explore the combination of IA with STBCs in different interference-limited scenarios. In fact, there is a tradeoff between rate, spatial diversity through space-time coding, and interference alignment, which has been fully characterized for the $K$-user multiple-input multipleoutput (MIMO) interference channel in [5]. A scheme that combines Alamouti codes and IA for the 2-user X channel where each user has 2 transmit and 2 receive antennas has been proposed in [6], and later extended to 4 antennas in [7]. In [8], the authors proposed a scheme that combines Alamouti codes with IA for the 3-user $2 \times 2$ MIMO interference channel. This method, however, requires a unidirectional cooperative link between two of the receivers in order to perform interference cancellation at the receiver where the interferences do not overlap, which might not always be feasible. In another line of work, [9] presented a scheme for zero-forcing or minimum mean-square error (MMSE) interference cancellation with Alamouti signals. This scheme requires at least as many receive antennas as users so that the interferences from unintended transmitters can be perfectly suppressed at the receiver side.

In this work we build on the representation of Alamouti space-time codewords as quaternions to combine Alamouti coding with interference alignment (IA) techniques. The inherent algebraic structure in orthogonal space-time block codes designs based on quaternions was already recognized in the early works on this field [10], [11], and since then it has been exploited in many different ways. We can mention, for instance, the design of full-diversity STBCs with rate higher than 1 in [12], or the design of a rate-2 differential STBC in [13]. A more general treatment of this topic can be found in [14]. It is also noteworthy the work in [15], where the authors formulated the multiuser detection of Alamouti signals as a MIMO detection problem in the quaternion domain. Following this line of work, here we formulate the IA problem with Alamouti signals using quaternions. By doubling the number of transmit antennas, we show that any single-stream feasible IA system in the complex domain can be transformed into an equivalent 
quaternionic IA system transmitting Alamouti signals and hence with increased diversity. To find interference alignment (IA) solutions for the equivalent quaternionic system, we reformulate in the quaternion domain the maximum signal-to-interference-plus-noise ratio (MaxSINR) algorithm proposed in [2].

\section{A. Notation}

In this paper, we will use boldfaced uppercase letters to denote matrices, boldfaced lowercase letters for column vectors, and lightfaced lowercase for scalar quantities. Quaternion scalars, vectors or matrices will be denoted with superscript $(\cdot)^{q}: x^{q}, \mathbf{x}^{q}$ or $\mathbf{X}^{q}$; and $\mathfrak{R}(\cdot)$ and $\mathfrak{I}(\cdot)$ denote the real and imaginary parts of a complex number. The superscripts $(\cdot)^{*},(\cdot)^{T}$ and $(\cdot)^{H}$ denote quaternion (or complex) conjugate, transpose and Hermitian, respectively. $\mathbf{I}_{N}^{q}$ is the quaternion identity matrix of dimensions $N \times N$.

\section{Alamouti CODING IN QUATERNIONIC FORM}

The Alamouti STBC (space-time block-coding) with 2 transmit antennas and one receive antenna groups the input symbols into pairs, $(s[n], s[n+1])$, and transmits the following codeword [4]

$$
\mathbf{S}[n]=\left(\begin{array}{cc}
s[n] & s[n+1] \\
-s^{*}[n+1] & s^{*}[n]
\end{array}\right) \quad \begin{array}{cc}
\downarrow & \text { time } \\
\text { space }
\end{array}
$$

The received signal over two consecutive time slots can be written as

$$
\begin{aligned}
& \left(\begin{array}{cc}
y[n] & y[n+1] \\
-y^{*}[n+1] & y^{*}[n]
\end{array}\right)=\left(\begin{array}{cc}
h(1) & h(2) \\
-h^{*}(2) & h^{*}(1)
\end{array}\right) \\
& \left(\begin{array}{cc}
s[n] & s[n+1] \\
-s^{*}[n+1] & s^{*}[n]
\end{array}\right)+\left(\begin{array}{cc}
r[n] & r[n+1] \\
-r^{*}[n+1] & r^{*}[n]
\end{array}\right),
\end{aligned}
$$

where $(y[n], y[n+1])$ is the received signal, $(r[n], r[n+1])$ is the additive white Gaussian noise (AWGN) with variance $\sigma^{2}$, and $h(1), h(2)$ are the complex channel gains from transmit antennas one and two to the receive antenna, respectively. We assume a flat-fading Rayleigh channel that remains fixed over a frame of $l$ consecutive Alamouti codewords and changes independently from frame to frame (block fading channel).

The $2 \times 2$ complex matrices in Eq. (2) represent an embedding of the quaternion algebra. Quaternions form a noncommutative division algebra $\mathbb{H}$, i.e., for $x^{q}, y^{q} \in \mathbb{H}$, $x^{q} y^{q} \neq y^{q} x^{q}$ in general. The conjugate of a quaternion $x^{q}=a+i b+j c+k d$ is $\left(x^{q}\right)^{*}=a-i b-j c-k d$, and the quaternion norm is defined as $\left|x^{q}\right|=\sqrt{x^{q}\left(x^{q}\right)^{*}}=$

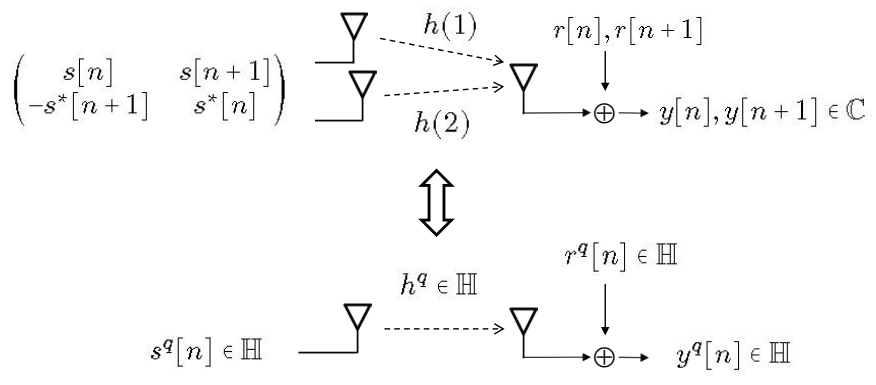

Fig. 1. A $2 \times 1$ complex MISO system with Alamouti coding, and its equivalent quaternionic SISO system (which involves two complex symbols and two complex channel uses).

$\sqrt{a^{2}+b^{2}+c^{2}+d^{2}}$. A more detailed treatment of quaternions can be found in [16]. For results regarding matrices of quaternions and the statistical analysis of quaternion random vectors we refer the reader to [17] and [18], respectively.

Using the Cayley-Dickson representation, the transmitted Alamouti codeword can alternatively be expressed as the following quaternion

$$
s^{q}[n]=\overbrace{\Re(R[n])+i \Im(s[n])}^{s[n]}+\overbrace{j \Re(s[n+1])+k \mathfrak{I}(s[n+1])}^{s[n+1] j}
$$

where we have applied the well-known properties of the quaternion imaginary units $(i, j, k)$. The other quaternions in Eq. (2) for the channel, the noise and the received signal can be defined likewise, and therefore the received signal over two time slots in (2) can be written more compactly as a single-input single-output (SISO) quaternion system

$$
y^{q}[n]=h^{q} s^{q}[n]+r^{q}[n],
$$

where now all variables $y^{q}[n], s^{q}[n], h^{q}, r^{q}[n] \in \mathbb{H}$ are scalar quaternions.

In summary, for the purpose of this paper the main point to realize is that a $2 \times 1$ multiple-input single-output (MISO) system transmitting Alamouti encoded signals is equivalent to a SISO system in quaternion form. This idea is depicted in Fig. 1.

\section{INTERFERENCE ALIGNMENT WITH AlAmouti CODED SIGNALS}

Consider the $K$-user interference channel with each transmitter having $M=2$ antennas and each receiver having $N=K-1$ antennas. Each user wishes to send $d=1$ streams or messages. We adhere to the notation used in [19] and denote this symmetric fully-connected network as $(2 \times(K-1), 1)^{K}$. This system is known to 
be feasible [19], [20], and thus we can perfectly suppress the interferences due to all undesired transmitters in the network by means of interference alignment (IA) [1]. This translates into a sum multiplexing gain (also known as degrees of freedom or DoF) of $\mathrm{K}$ for this network, which for the $K=3$ user case is known to be optimal. However, as proved in [21], IA does not provide any spatial diversity gain in this single-stream network. In other words, using perfect or zero-forcing IA for the $(2 \times 2,1)^{3}$ network we achieve $3 \mathrm{DoF}$ but the diversity gain is only 1 .

Alamouti STBC is a simple linear combining technique to achieve spatial diversity for links with 2 transmit antennas [4]. Although this scheme was later generalized to $M$ transmit antennas in [22] (but with a reduction in multiplexing gain), in this paper we will focus on the Alamouti scheme due to its simplicity and widespread use.

A simple way to combine the benefits of IA (in terms of DoF) and Alamouti coding (in terms of spatial diversity) is to double the number of transmit antennas and then exploit the quaternion formulation described in Section II. The basic idea is that a $(4 \times(K-1), 1)^{K}$ system transmitting Alamouti signals is equivalent to a $(2 \times(K-1), 1)^{K}$ network with quaternion variables to which the conventional IA algorithms and solutions can be applied after an appropriate reformulation in the quaternion domain. Specifically, for a $(4 \times(K-1), 1)^{K}$ system transmitting Alamouti signals, let $\mathbf{v}_{k}^{q} \in \mathbb{H}^{2 \times 1}$ be the unitary quaternion precoder for user $k$ and let $s_{k}^{q} \in \mathbb{H}$ be the STBC signal transmitted by user $k$; then, the signal at receiver $k$ is given by (we skip the time index $n$ for notational simplicity)

$$
\mathbf{y}_{k}^{q}=\mathbf{H}_{k, k}^{q} \mathbf{v}_{k}^{q} s_{k}^{q}+\sum_{l \neq k} \mathbf{H}_{k, l}^{q} \mathbf{v}_{l}^{q} s_{l}^{q}+\mathbf{n}_{k}^{q},
$$

where $\mathbf{H}_{k, l}^{q} \in \mathbb{H}^{(K-1) \times 2}$ is the flat fading quaternion MIMO channel from transmitter $l$ to receiver $k$, and $\mathbf{n}_{k} \in \mathbb{H}^{(K-1) \times 1}$ is the AWGN at the $k$-th receiver.

Let $\mathbf{u}_{k}^{q} \in \mathbb{H}^{(K-1) \times 1}$ be a unit-norm quaternion vector representing the linear receiver for user $k$. Solving the IA problem amounts to finding a set of precoders and decoders satisfying the following conditions

$$
\begin{gathered}
\left(\mathbf{u}_{k}^{q}\right)^{H} \mathbf{H}_{k l}^{q} \mathbf{v}_{l}^{q}=0, \quad \forall k \neq l, \\
\left(\mathbf{u}_{k}^{q}\right)^{H} \mathbf{H}_{k k}^{q} \mathbf{v}_{k}^{q} \neq 0, \quad \forall k,
\end{gathered}
$$

where (6) guarantees that all interferences are properly zero-forced, while (7) preserves the desired link at the intended receivers. After applying the IA precoder and decoder, we obtain an equivalent quaternion SISO system

$$
z_{k}^{q}=\overbrace{\left(\mathbf{u}_{k}^{q}\right)^{H} \mathbf{H}_{k k}^{q} \mathbf{v}_{k}^{q}}^{h_{e q}^{q}, k} s_{k}^{q}+\overbrace{\left(\mathbf{u}_{k}^{q}\right)^{H} \mathbf{n}_{k}^{q}}^{r_{k}^{q}}=h_{e q, k}^{q} s_{k}^{q}+r_{k}^{q},
$$

where we have applied (6) to cancel the interferences from unintended transmitters, and $r_{k}^{q}$ is AWGN with variance $\sigma^{2}$. The maximum likelihood (ML) decoder is based on the search for the closest (quaternion) constellation point to the estimate $\left(h_{e q, k}^{q}\right)^{*} z_{k}^{q} /\left|h_{e q, k}^{q}\right|^{2}$.

In the complex case, closed-form IA solutions are known only for certain scenarios like the $(2 \times 2,1)^{3}$ interference channel, for which the precoder for user 1 , $\mathbf{v}_{1}$, is an eigenvector of the matrix $\mathbf{E} \in \mathbb{C}^{2 \times 2}$ given by

$$
\mathbf{E}=\mathbf{H}_{31}^{-1} \mathbf{H}_{32} \mathbf{H}_{12}^{-1} \mathbf{H}_{13} \mathbf{H}_{23}^{-1} \mathbf{H}_{21},
$$

and then the precoders for users 2 and 3 are obtained as $\mathbf{v}_{2}=\mathbf{H}_{32}^{-1} \mathbf{H}_{31} \mathbf{v}_{1}$ and $\mathbf{v}_{3}=\mathbf{H}_{23}^{-1} \mathbf{H}_{21} \mathbf{v}_{1}$, respectively. Unfortunately, the noncommutative multiplication of quaternions introduce some obstacles when working with quaternion matrices that prevent us from directly applying this closed-form solution to the $(2 \times 2,1)^{3}$ quaternion system. The main problem being that there is no general theory of eigenvalues and eigenvectors for arbitrary quaternion matrices [23]. In a remarkable work, however, Le Bihan and Mars extended the singular value decomposition algorithm (SVD) to the skew algebra of quaternions [18]. This quaternionic SVD allows us to extend the two distributed iterative algorithms proposed in [2], namely, the interference leakage minimization algorithm that perfectly suppress the interference from all undesired transmitters, and the maximum signal-tointerference-plus-noise-ratio (Max-SINR) algorithm that maximizes the output SINR at each receiver. For completeness, we include in Algorithm 1 the steps of the Max-SINR algorithm in [2] with quaternion variables. Furthermore, these algorithms can be applied to any other feasible scenario with more than 3 users.

Remark 1. The feasibility of IA problems with quaternion variables would require a more detailed study, because the algebraic geometry tools used in the complex case [19], [20] do not directly translate to the skew algebra of quaternions. Nevertheless, it has been observed experimentally that the quaternion-based interference leakage minimization algorithm converges to a perfectly aligned solution whenever the complex system with the same dimensions is feasible. 


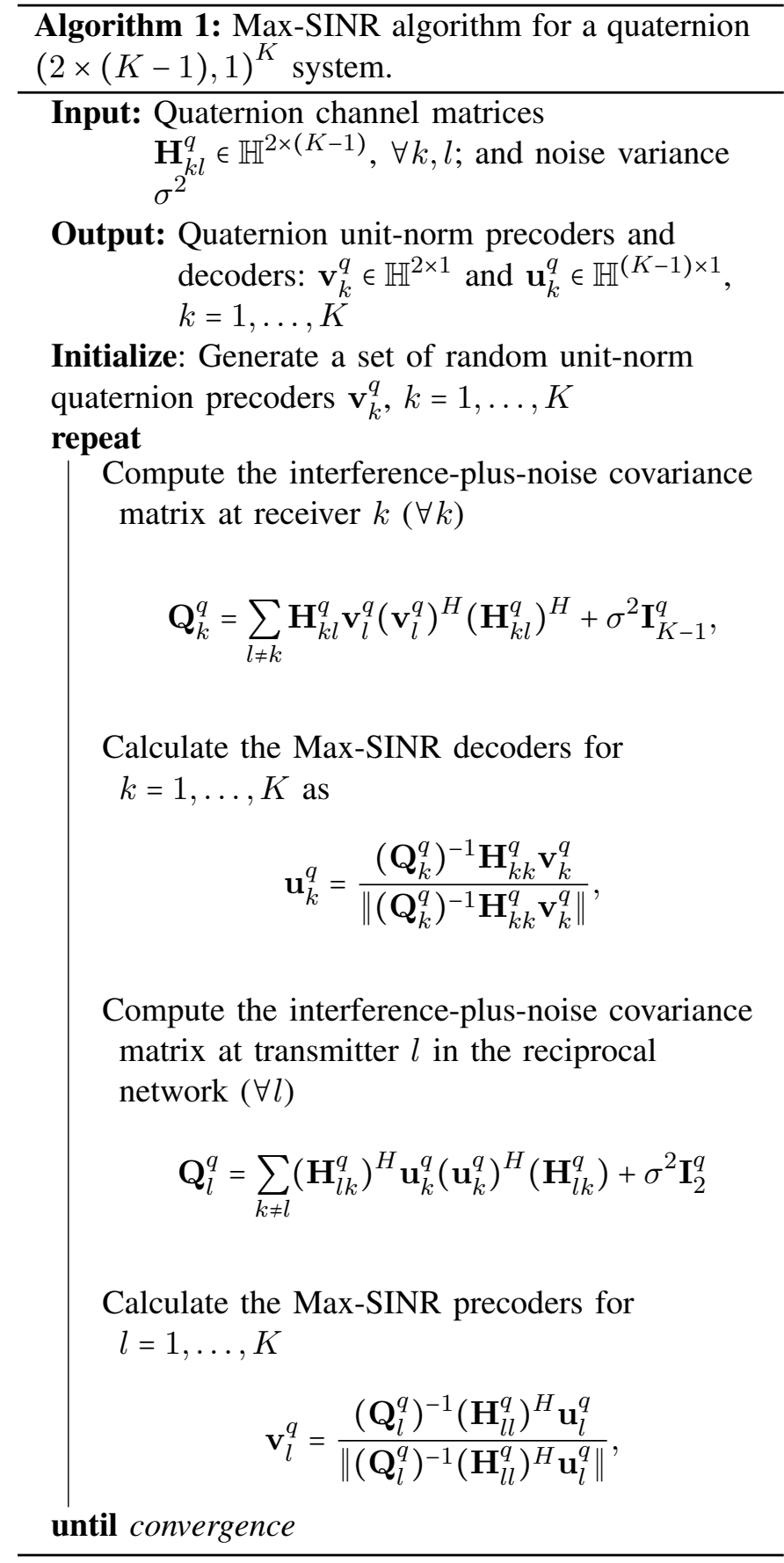

\section{Simulation Results}

In this section we compare the performance of the quaternionic IA+Alamouti algorithm with some competing schemes in the complex number domain with and without Alamouti coding. We consider a 3-user MIMO interference channel and assume perfect CSI for all schemes. The data symbols are assumed to be uncoded 4-QAM symbols generated independently at every transmitter. Using this fixed-rate modulation, the spectral efficiently for the $K$-user channel is $2 K$ bits per channel use (bpcu). The average symbol error rate (SER) has been estimated for 500 independent Rayleigh channel realizations through Monte Carlo simulations. For each channel realization and for each signal-tonoise-ratio (SNR) we transmitted a sequence of $1 e 6$ symbols. For the quaternionic implementation of the Max-SINR algorithm we use the open-source Matlab® toolbox developed by Sangwine and Le Bihan [24].

Fig. 2 shows the SER curves for user 1 (the behavior for other users is similar) as a function of the average signal-to-noise-ratio $(\mathrm{S} N R)$ per receive antenna, which is defined as $\mathrm{S} N R=10 \log _{10}\left(1 / \sigma^{2}\right)$. As a baseline for performance, we include in the comparison a $(2 \times 2)$ IA scheme in the complex domain without Alamouti encoding and thus with no diversity gain [21]. We also include in the comparison a 3 -user $(2 \times 3)$ system using the Max-SINR IA algorithm in the complex domain and without Alamouti coding which, as shown in [21], achieves a diversity gain of 2 . As shown in [9], [25], the same diversity gain can be achieved in the $(2 \times 3)$ system when the transmitters send Alamouti signals and the receivers apply the minimum mean-square error (MMSE) decoder to suppress the interference. Interestingly, this scheme can be formulated in the complex domain or in the quaternion domain as shown in [15] with similar performance. This method is labeled in Fig. 2 as ICMMSE+Alamouti. Finally, for the proposed quaternionic IA-Alamouti scheme each user has $M=4$ antennas at the transmitter and $N=2$ at the receiver. This method also achieves a diversity gain of 2 but with some additional SNR gain because of the extra antenna at the transmit side.

\section{Conclusions}

This paper proposed a scheme to combine interference alignment (to realize the maximum achievable DoF of the system) with Alamouti coding (to provide diversity gain) for fixed-rate constellations. Using a formulation based on quaternions, we showed that the space-time encoding/decoding and the IA precoding/decoding operations can be performed independently. This separation between coding for diversity and aligning for interference suppression, although not necessarily optimal, allows for the independent optimization of the IA precoders and decoders using alternating optimization algorithms. In its simplest realization, each user uses two antennas for alignment and two antennas for Alamouti encoding. Although in terms of performance there is no inherent advantage of the quaternion domain over the 


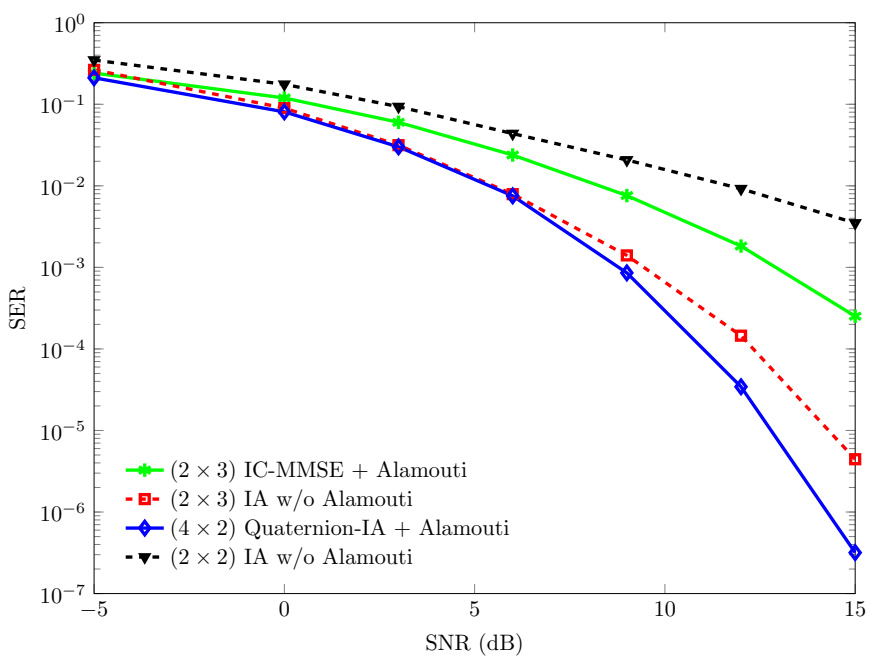

Fig. 2. Performance comparison of interference alignment and interference cancellation techniques with and without Alamouti signals for a 3-user MIMO interference channel.

complex number domain, we believe that the quaternion formulation proposed in this paper opens up a new perspective for the analysis and optimization of systems combining Alamouti coded signals with interference alignment or interference suppression techniques.

\section{ACKNOWLEDGMENT}

This work has been supported by the Ministerio de Economa, Industria y Competitividad (MINECO) of Spain, under grants TEC2013-47141-C4-R (RACHEL), TEC201675067-C4-4-R (CARMEN), and FPI grant BES-2014-069786.

\section{REFERENCES}

[1] V. R. Cadambe and S. A. Jafar, "Interference alignment and degrees of freedom of the K-user interference channel," IEEE Trans. Inf. Theory, vol. 54, no. 8, pp. 3425-3441, 2008.

[2] K. S. Gomadam, V. R. Cadambe, and S. A. Jafar, "A distributed numerical approach to interference alignment and applications to wireless interference networks," IEEE Trans. Inf. Theory, vol. 57, no. 6, pp. 3309-3322, Jun. 2011.

[3] I. Santamaria, Ó. González, R. W. Heath, and S. W. Peters, "Maximum sum-rate interference alignment algorithms for MIMO channels," in Proc. IEEE Global Telecomm. Conference (GLOBECOM 2010), Miami, FL, USA, Dec. 2010, pp. 1-6.

[4] S. Alamouti, "A simple transmit diversity technique for wireless communications," IEEE J. Select. Areas Commun., vol. 16, no. 8, pp. 1451-1458, Oct. 1998.

[5] A. Sezgin, S. A. Jafar, and H. Jafarkhani, "Optimal use of antennas in interference networks: a tradeoff between rate, diversity and interference alignment," in Proc. IEEE Global Telecomm. Conf. (GLOBECOM 2009), 2009.

[6] L. Li, H. Jafarkhani, and S. A. Jafar, "When Alamouti codes meet interference alignment: transmission schemes for two-user $\mathrm{X}$ channel," in Proc. IEEE Int. Symposium Inf. Theory (ISIT 2011), Jul. 2011, pp. 2577-2581.
[7] A. Ganesan and B. Sundar Rajan, "Interference alignment with diversity for the $2 \times 2 \mathrm{X}$-network with four antennas," IEEE Trans. Inf. Theory, vol. 60, no. 6, pp. 3576-3592, 2014.

[8] A. Zaki, C. Wang, and L. K. Rasmussen, "Combining interference alignment and Alamouti codes for the 3-user MIMO interference channel," in IEEE Wireless Comm. and Networking Conf., 2013, pp. 3563-3567.

[9] A. F. Naguib, N. Seshadri, and A. R. Calderbank, "Applications of space-time block codes and interference suppression for high data rate wireless systems," in Proc. Asilomar Conf. on Signals, Systems and Computers, 1998, pp. 1803-1810.

[10] V. Tarokh, H. Jafarkhani, and A. R. Calderbank, "Space-time block codes from orthogonal designs," IEEE Trans. Inf. Theory, vol. 45, no. 5, pp. 1456-1467, 1999.

[11] B. A. Sethuraman, B. Sundar Rajan, and V. Shashidhar, "Fulldiversity, high-rate space-time block codes from division algebras," IEEE Trans. Inf. Theory, vol. 49, no. 10, pp. 2596-2616, 2003

[12] S. Das, N. Al-Dhahir, and R. Calderbank, "Novel full-diversity high-rate STBC for 2 and 4 transmit antennas," IEEE Comm. Letters, vol. 10, no. 3, pp. 1-3, 2006.

[13] N. Al-Dhahir, "A new high-rate differential space-time block coding scheme," IEEE Comm. Letters, vol. 11, no. 11, pp. 540542, 2003.

[14] J. Seberry, K. Fynlayson, S. S. Adams, A. Wysocki, T. Xia, and B. J. Wysocki, "The theory of quaternion orthogonal designs," IEEE Trans. Sig. Process., vol. 56, no. 1, pp. 256-265, 2008.

[15] C. W. Tan and A. R. Calderbank, "Multiuser detection of Alamouti signals," IEEE Trans. Comm., vol. 57, no. 7, pp. 1-10, 2009.

[16] J. P. Ward, Quaternions and Cayley Numbers: Algebra and Applications. Dordrecht, The Netherlands: Kluwer, 1997.

[17] J. Via, D. Ramirez, and I. Santamaria, "Properness and widely linear processing of quaternion random vectors," IEEE Trans. Inf. Theory, vol. 56, no. 7, pp. 3502-3515, Jul. 2010.

[18] N. Le Bihan and J. Mars, "Singular value decomposition of quaternion matrices: A new tool for vector-sensor signal processing," Signal Processing, vol. 84, no. 7, pp. 1177-1199, Jul. 2004.

[19] C. M. Yetis, T. Gou, S. A. Jafar, and A. H. Kayran, "On feasibility of interference alignment in MIMO interference networks," IEEE Trans. Sig. Process., vol. 58, no. 9, pp. 47714782, Sep. 2010.

[20] Ó. González, C. Beltrán, and I. Santamaria, "A feasibility test for linear interference alignment in MIMO channels with constant coefficients," IEEE Trans. Inf. Theory, vol. 60, no. 3, pp. 1840-1856, Mar. 2014.

[21] H. Ning, C. Ling, and K. K. Leung, "Feasibility condition for interference alignment with diversity," IEEE Trans. Inf. Theory, vol. 57, no. 5, pp. 2902-2912, May 2011.

[22] V. Tarokh, H. Hafarkhani, and A. R. Calderbank, "Space-time block codes from orthogonal designs," IEEE Trans. Inf. Theory, vol. 45, no. 5, pp. 1456-1467, Jul. 1999.

[23] F. Zhang, "Quaternions and matrices of quaternions," Linear Algebra and its Applications, vol. 257, no. 5, pp. 21-57, Jan. 1997.

[24] S. J. Sangwine and N. Le Bihan, "Quaternion toolbox for Matlab®. software library available at: http://qtfm.sourceforge.net/," 2013.

[25] A. Stamoulis, N. Al-Dhahir, and A. R. Calderbank, "Further results on interference cancellation and space-time block codes," in Proc. Asilomar Conf. on Signals, Systems and Computers, 2001, pp. 257-261. 